\title{
Relativistic hybrid stars in light of the NICER PSR J0740+6620 radius measurement
}

\author{
Jia Jie $\mathrm{Li}^{*}$ \\ School of Physical Science and Technology, Southwest University, Chongqing 400700, China \\ Armen Sedrakian \\ Frankfurt Institute for Advanced Studies, D-60438 Frankfurt am Main, Germany and \\ Institute of Theoretical Physics, University of Wroclaw, 50-204 Wroclaw, Poland \\ Mark Alford \\ Department of Physics, Washington University, St. Louis, Missouri 63130, USA
}

\begin{abstract}
We explore the implications of the recent radius determination of PSR J0740+6620 by the NICER experiment combined with the neutron skin measurement by the PREX-II experiment and the associated inference of the slope of symmetry energy, for the structure of hybrid stars with a strong first-order phase transition from nucleonic to quark matter. We combine a covariant density-functional nucleonic equation of state (EOS) with a constant-speed-of-sound EOS for quark matter. We show that the radius and tidal deformability ranges obtained from GW170817 can be reconciled with the implication of the PREX-II experiment if there is a phase transition to quark matter in the low-mass compact star. In the high-mass segment, the EoS needs to be stiff to comply with the large-radius inference for PSR J0740+6620 and J0030+0451 with masses $M \simeq 2 M_{\odot}$ and $M \simeq 1.4 M_{\odot}$. We show that twin stars are not excluded, but the mass and radius ranges (with $M \geq M_{\odot}$ ) are restricted to narrow domains $\Delta M_{\text {twin }} \lesssim 0.05 M_{\odot}$ and $\Delta R_{\text {twin }} \sim 1.0 \mathrm{~km}$. We also show that the existence of twin configurations is compatible with the light companion in the GW190814 event being a hybrid star in the case of values of the sound-speed square $s=0.6$ and $s=1 / 3$.
\end{abstract}

\section{INTRODUCTION}

The recent mass measurement of the heaviest pulsar to date PSR J0740+6620 [1], which is currently at $2.08_{-0.07}^{+0.07} M_{\odot}$ and the recent analysis $[2,3]$ of $x$-ray data from the NICER experiment, which gave the radius estimates $12.39_{-0.98}^{+1.30}$ [2] and $13.71_{-150}^{+2.61} \mathrm{~km} \mathrm{[3]} \mathrm{and} \mathrm{corresponding} \mathrm{mass} \mathrm{estimates}$ $2.07_{-0.07}^{+0.07} M_{\odot}$ and $2.08_{-0.09}^{+0.09} M_{\odot}$ of PSR J0740+6620 open prospects of constraining the properties of dense-matter equation of state (EOS) - in particular, the possibility of a phase transition at high densities.

The lower bound on the radius of PSR J0740+6620 constrains a highly relevant region of the mass-radius $(M-R)$ diagram of compact stars (CSs), which in combination with the determination of the tidal deformability (TD) of a star of mass $\sim 1.4 M_{\odot}$ in the GW170817 event by the LIGO-Virgo Collaboration [4], puts significant constraints on the EOSs of CSs. These require that the stellar EOS must be moderately soft at intermediate densities (to allow for relatively small TDs) and must be stiff enough at high densities (to allow for two-solarmass CSs; see Refs. [5-11]).

Recently, the Lead Radius Experiment Collaboration (PREX-II) reported the most precise measurement yet of the neutron skin thickness of the lead nucleus $R_{\text {skin }}^{208}=0.283 \pm$ $0.071 \mathrm{fm}$ (mean and $1 \sigma$ standard deviation), in a parityviolating electron scattering experiment [12]. Subsequent theoretical analysis $[13,14]$ based on density-functional theory established values of the symmetry energy $E_{\text {sym }}$ and the

\footnotetext{
*jiajieli@swu.edu.cn

$\dagger$ sedrakian@fias.uni-frankfurt.de

¥ alford@physics.wustl.edu
}

slope of nuclear symmetric energy $L_{\mathrm{sym}}$ at saturation density $\left(\rho_{\text {sat }}\right)$ that are consistent with the inferred value of $R_{\text {skin }}^{208}$. Reference [13] finds $E_{\text {sym }}=38.1 \pm 4.7 \mathrm{MeV}$ and $L_{\text {sym }}=$ $106 \pm 37 \mathrm{MeV}$ from a family of relativistic (nonlinear, mesonexchange) density functionals (DFs). Reference [14] expanded the base (and the functional form) of employed DFs to include nonrelativistic DFs, relativistic DFs with densitydependent meson-exchange couplings and relativistic point coupling DFs to find $E_{\text {sym }}=32 \pm 1 \mathrm{MeV}$ and $L_{\text {sym }}=54 \pm 8$ $\mathrm{MeV}$. These values include the additional requirement on DFs to be consistent with the experimental limits on the dipole polarizability of ${ }^{208} \mathrm{~Pb}$, which prefer DFs predicting a small value of $L_{\mathrm{sym}}$. The large value of $L_{\mathrm{sym}}$ found in the first analysis is in potential tension with the various estimates [15-18], whereas the value obtained from the second analysis is within the range inferred previously. Note that the difference between the two quoted values of $L_{\text {sym }}$ is $1.37 \sigma$ which translates to about $83 \%$ significance. Since $L_{\mathrm{sym}}$ is highly correlated with the stellar radius and TD, the rather large value found in the first analysis is in potential tension with the GW170817 deformability measurement if one assumes a purely nucleonic composition [13].

Nevertheless, independent of the interpretation of the PREX-II experiment, the potential tension outlined above suggests a close examination of the compatibility of a large value of $L_{\text {sym }}$ with the constraints on compact stars. Here we address the possibility of first-order phase transition from nucleonic to quark matter using (a) the radius measurements of two neutron stars by NICER, (b) the TD of GW170817, and (c) a range of values of $L_{\mathrm{sym}}$ suggested by Refs. [13, 14].

The composition of matter at high densities achieved in a CS's core remains unknown. One possibility is a deconfinement phase transition from bound states of hadrons to liberated quark states [19]. If the quark matter in a CS's core is relatively soft then its radius and TD are reduced, which could 


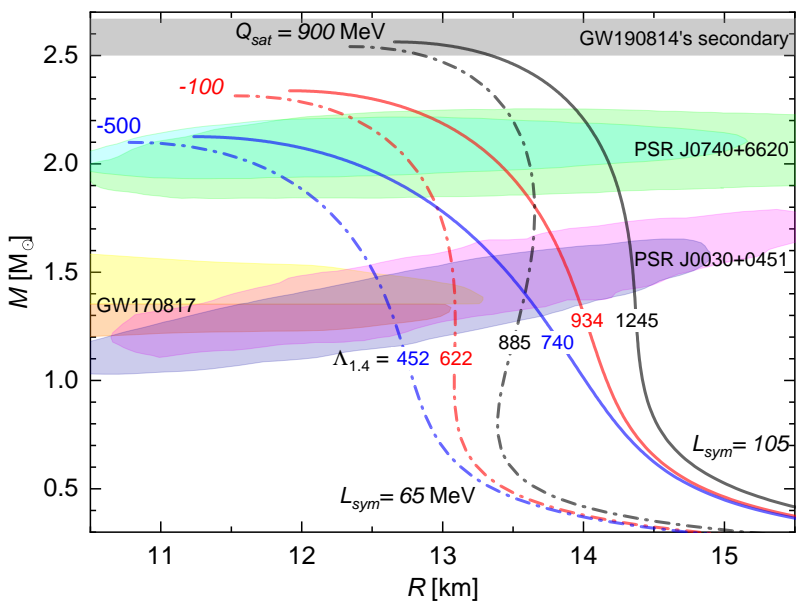

FIG. 1. $M-R$ relation for nucleonic EOS for different pairs of values of $Q_{\text {sat }}$ and $L_{\text {sym }}$. In addition we show the TD of a canonical CS for each model. Constraints with $90 \%$ credibility from multimessenger astronomy are shown by shaded regions [2-4, 37, 38]; see text for details.

avoid the tentative tension between the inferred TDs from the GW170817 event and those predicted by purely hadronic stiff EOS models without a phase transition [20-34]. Of particular interest in this scenario is the emergence of twin and triplet stars, where one (or two) hybrid stars have the same mass as, but a different radius from, a purely hadronic star [21, 25$27,30,35,36]$. The more extreme case of triplets will not be discussed here.

With a mass of about $2.1 M_{\odot}$, PSR J0740+5620 is the most massive known CS: it is about 50\% more massive than PSR J0030+0451. Yet current measurements do not indicate a significant difference in size; see Fig. 1. As mentioned earlier, the generic feature of the first-order phase transition is the softening of the EOS in the vicinity of the transition point, which leads to smaller radii of hybrid stars as compared to the nucleonic ones. Confronting the very massive star's radius estimation with the possible phase transition in dense matter is thus very timely. This issue has been discussed by Refs. [5, 7].

In this work, we apply the setup developed by us earlier [30] to explore the consequences of a strong first-order phase transition from nucleonic to quark matter and the formation of twin stars. The resulting models are then confronted with the newly available data from NICER and PREX-II experiments.

\section{CONSTRUCTION OF EOS}

To describe low-density matter, we use a covariant density functional (CDF) of nuclear matter in which baryons are coupled to mesons with density-dependent couplings, and we adopt as a reference the DDME2 parametrization [39]. The CDF EOS models can be extended by modifying the density dependence of the couplings [40] such as to map the CDF EOS models onto purely phenomenological ones [41], which are based on an expansion of the energy density of nuclear matter close to the saturation density $\rho_{\text {sat }}$ with respect to the density and isospin asymmetry-i.e.,

$$
\begin{aligned}
E(\chi, \delta) \simeq & E_{\mathrm{sat}}+\frac{1}{2 !} K_{\mathrm{sat}} \chi^{2}+\frac{1}{3 !} Q_{\mathrm{sat}} \chi^{3} \\
& +E_{\mathrm{sym}} \delta^{2}+L_{\mathrm{sym}} \delta^{2} \chi+O\left(\chi^{4}, \chi^{2} \delta^{2}\right),
\end{aligned}
$$

where $\chi=\left(\rho-\rho_{\text {sat }}\right) / 3 \rho_{\text {sat }}$ with $\rho$ and $\rho_{\text {sat }}$ being the number density and its value at saturation, and $\delta=\left(\rho_{n}-\rho_{p}\right) / \rho$ where $\rho_{n(p)}$ is the neutron(proton) number density. The coefficients of the expansion are known as the incompressibility $K_{\text {sat }}$, the skewness $Q_{\text {sat }}$, the symmetry energy $E_{\mathrm{sym}}$, and the slope parameter $L_{\mathrm{sym}}$. In this manner, the uncertainties in the gross properties of CSs can be quantified in terms of not-wellknown higher-order characteristics of nuclear matter, specifically $Q_{\text {sat }}$ and $L_{\text {sym }}[40,41]$. The low-order characteristics are fixed at their values predicted by the DDME2 parametrization: $E_{\text {sat }}=-16.14, K_{\text {sat }}=251.15, E_{\mathrm{sym}}=32.31 \mathrm{MeV}$ and $\rho_{\text {sat }}=0.152 \mathrm{fm}^{-3}$. At the same time, the CDF provides access to the composition of matter which is not fixed in agnostic models based on nuclear characteristics only.

Motivated by this, we construct six representative EOSs featuring combinations of three values of $Q_{\text {sat }}=-500$, $100,900 \mathrm{MeV}$ and two values of $L_{\mathrm{sym}}=65,105 \mathrm{MeV}$. The value of $Q_{\text {sat }}$ controls the high-density behavior of the EOS, and thus, the maximum mass of a static CS [40]. For $Q_{\text {sat }}=-500 \mathrm{MeV}$ the maximum mass is about $2 M_{\odot}$, which matches the mass measurement of PSR J0740+6620 [1, 42]; for $Q_{\text {sat }}=-100 \mathrm{MeV}$ the maximum mass is consistent with the (approximate) upper limit on the maximum mass of static CSs 2.3 $M_{\odot}$ inferred from the analysis of GW170817 event [43, 44]; finally, for $Q_{\text {sat }}=900 \mathrm{MeV}$ the maximum mass is close to $2.5 M_{\odot}$, which would be compatible with the mass of the secondary in the GW190814 event [45] and its interpretation as a nucleonic CS $[46,47]$. We chooe values of $L_{\text {sym }}$ corresponding to the central value and the lower range of the $1 \sigma$ confidence interval (CI) of the PREX-II measurement, $L_{\text {sym }}=106 \pm 37 \mathrm{MeV}[12,13]$. Note that the recent measurement of the spectra of pions in intermediate energy collisions implies $L_{\mathrm{sym}}=79.9 \pm 37.6 \mathrm{MeV}$ [48]. In addition, an analysis based on nonparametric EoS shows that there is a mild tension between the results of Ref. [13] and astrophysical data supplemented by chiral effective field theory results [49].

The $M-R$ relations for our six EOS are shown in Fig. 1, where we also show the current astrophysical observational constraints. We show $90 \%$-credible ellipses from each of the two NICER modeling groups for PSR J0030+0451 and J0740+6620 [2, 3, 37, 38]. We also show 90\%-credible regions for each of the two CSs that merged in the gravitational wave event GW170817 [4]. Finally, we show the 90\% CI for the mass of the secondary component of GW190814 [45]. A lower limit on the average TD $\tilde{\Lambda}_{1.186} \geq 240$ (with binary chirp mass $\mathcal{M}=1.186 M_{\odot}$ ) was extracted from the GW170817 event using the observations and modeling of this merger [50,51]. This limit allows for hybrid stars having $R_{1.4}$ radii [24] well below the corresponding NICER limits $[37,38]$.

The softest EOS model $\left(Q_{\text {sat }}=-500, L_{\text {sym }}=65 \mathrm{MeV}\right)$, which gives $R_{1.4}=12.58 \mathrm{~km}$ and $\Lambda_{1.4}=452$ for canonicalmass star, appears as the only model satisfying all three of the 
$M-R$ constraints. The stiffest EOS model $\left(Q_{\mathrm{sat}}=900, L_{\mathrm{sym}}=\right.$ $105 \mathrm{MeV}$ ) which gives $R_{1.4}=14.37 \mathrm{~km}$ and $\Lambda_{1.4}=1245$, passes through the upper range of the CIs provided by NICER results. The $M-R$ relations shown in Fig. 1 indicate that one could find a compromise between the requirements of a soft EoS (from GW170817) and relatively large value of $L_{\text {sym }}$ implied by the analysis of PREX-II in Ref. [13]. This would be accomplished by an EOS with $L_{\text {sym }} \sim 65 \mathrm{MeV}$, which is at the lower end of $1 \sigma$ range of Ref. [13], and a negative $Q_{\text {sat }}$.

So far, we have seen that fairly low values of $L_{\text {sym }}$ from the range consistent with the inference [13] from the PREXII experiment allow us to build models that are consistent with the known astronomical constraints. However, as we show next, larger values from the experimental range of $L_{\text {sym }}$ can be accommodated if a strong first-order phase transition to quark matter is allowed for. We will model below the EOS of the quark phase using the constant-sound-speed (CSS) parametrization $[35,52]$, which matches well with the predictions based on the Nambu-Jona-Lasinio (NJL) model computations which include vector repulsion $[53,54]$. We assume a first-order phase transition with a sharp boundary between the nucleonic and quark phases (which is the case when mixed phases are disfavored by surface tension and electrostatic energy costs [55]). The pressure is then given by [35, 52]

$$
p(\varepsilon)= \begin{cases}p_{\text {tran }}, & \varepsilon_{\text {tran }}<\varepsilon<\varepsilon_{\text {tran }}+\Delta \varepsilon \\ p_{\text {tran }}+s\left[\varepsilon-\left(\varepsilon_{\text {tran }}+\Delta \varepsilon\right)\right], & \varepsilon_{\text {tran }}+\Delta \varepsilon<\varepsilon\end{cases}
$$

where $p_{\text {tran }}$ is the transitional pressure with energy density $\varepsilon_{\text {tran }}, \Delta \varepsilon$ is the discontinuity, and $s$ is the square of the sound speed in the quark phase. The possible topologies of hybrid stars in the $M-R$ diagram based on CSS parametrization have been studied [35]. Of particular interest is the case where, by an appropriate choice of the parameters $p_{\text {tran }}, \Delta \varepsilon$, and $s$, there are two disconnected branches of stars: one with purely nucleonic and the other with hybrid stars. Such topology leads, in particular, to twin configurations where stars have identical masses but different radii [21, 25-27]. The more extreme case of three disconnected branches leads to the formation of triplets, which will not be studied here [36].

Figure 2 illustrates how such a transition to quark matter allows a nuclear EOS with a large $L_{\text {sym }}$ to be consistent with astrophysical constraints. We have fixed the nuclear EOS by choosing $L_{\text {sym }}=65$ and $Q_{\text {sat }}=900 \mathrm{MeV}$. We vary the CSS parameters of the quark-matter EOS to explore different sound speeds $s$, and by varying $\rho_{\text {tran }}$ and $\Delta \varepsilon_{\text {tran }}$ we can explore different values of the maximum masses $M_{\max }^{\mathrm{N}}$ and $M_{\max }^{\mathrm{Q}}$ on the nucleonic branch and hybrid branch respectively. In particular, each of the two same-color solid lines differ only by the value of $M_{\mathrm{max}}^{\mathrm{Q}}$.

The sequences shown in Fig. 2 are for $M_{\max }^{\mathrm{Q}} / M_{\odot}=2.1$, 2.3, 2.5 and $M_{\max }^{\mathrm{N}} / M_{\odot}=1.0,1.3$ (corresponding to transition densities $\left.\rho_{\text {tran }} / \rho_{\text {sat }}=1.81,2.05\right)$. We see that a stiff nucleonic EOS can be made compatible with the GW170817 constraint if there is a first-order transition to quark matter at densities $\rho_{\text {tran }} \lesssim 2 \rho_{\text {sat }}$.

Figure 2 shows, in addition, the sensitivity of results toward varying the value of $s$ for a specific case with fixed maximum

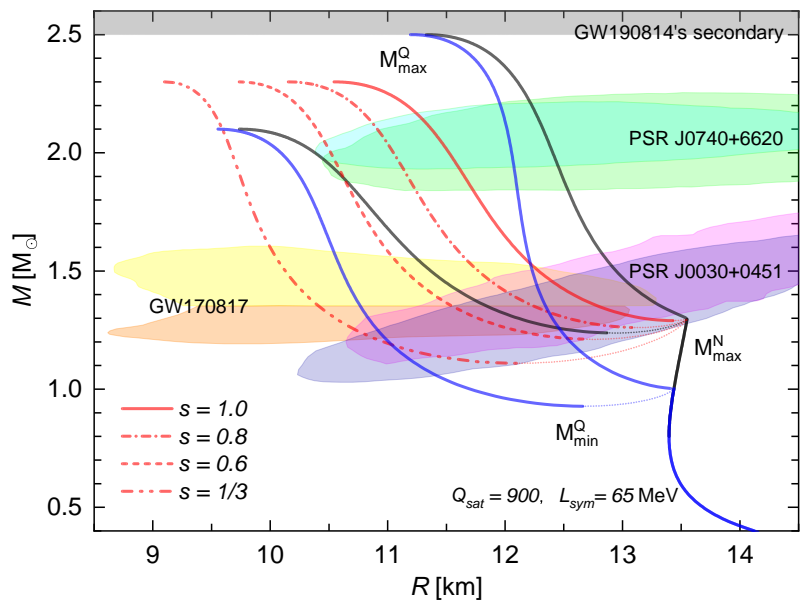

FIG. 2. Illustrative $M-R$ relation for hybrid EOS models. The results are constructed by varying one of the three quantities that fully determine the model at fixed values of the two others. These are the maximum masses of the quark $M_{\max }^{\mathrm{Q}}$ and nucleonic $M_{\max }^{\mathrm{N}}$ branches and the sound-speed square $s$. The dotted thin lines indicate unstable configurations. All the solid curves correspond to $s=1.0$. For the remaining curves, the value of $s$ is as indicated in the plot.

masses. Since a reduction of $s$ from its maximal value softens the quark-matter EOS, the $M-R$ curves are shifted to the left, eventually putting some of them $(s \lesssim 0.6)$ outside of the NICER result for PSR J0740+6620. Note that in Fig. 2 two topologies of $M-R$ curves are present: the connected and disconnected ones, the latter having a region of instability between the nucleonic and hybrid configurations. In the following, we focus on the disconnected topologies.

\section{MASS-RADIUS CONSTRAINT FOR HYBRID STARS}

To study the occurrence of twin configurations, we select EOSs from the parameter space of our model that have twin configurations and are consistent with both NICER and gravitational wave measurements. Specifically, they yield radii that are above the NICER 90\%-confidence lower limit from PSR J0740+6620 and J0030+0451, and below the 90\%confidence upper limit on the radius of a $1.36 M_{\odot}$ star from the GW170817 event; (the stellar mass is chosen to be the one inferred for an equal-mass binary.) Figure 3 shows examples of our exploration of this parameter space. Each of the six panels in Fig. 3 has curves for two nuclear EOS (with the values of $L_{\text {sym }}$ and $Q_{\text {sat }}$ given in each panel title) and a fixed speed of sound in the quark-matter EOS (also given in each panel title). Within each panel we vary the remaining parameters of the quark-matter EoS (nuclear matter density at the transition $\rho_{\text {tran }}$ and energy density jump at the transition $\Delta \varepsilon$ ) as follows: In each of the one or two plots in each panel, we require the maximum mass on the hybrid branch $M_{\max }^{\mathrm{Q}}$ to have a different value (which can be read off from the $y$ axis), and in each plot, we show results for two values of $M_{\mathrm{max}}^{\mathrm{N}}$ which span the range within which the $M-R$ curves obey the mass-radius constraints as described in the previous paragraph, and contain twin stars 

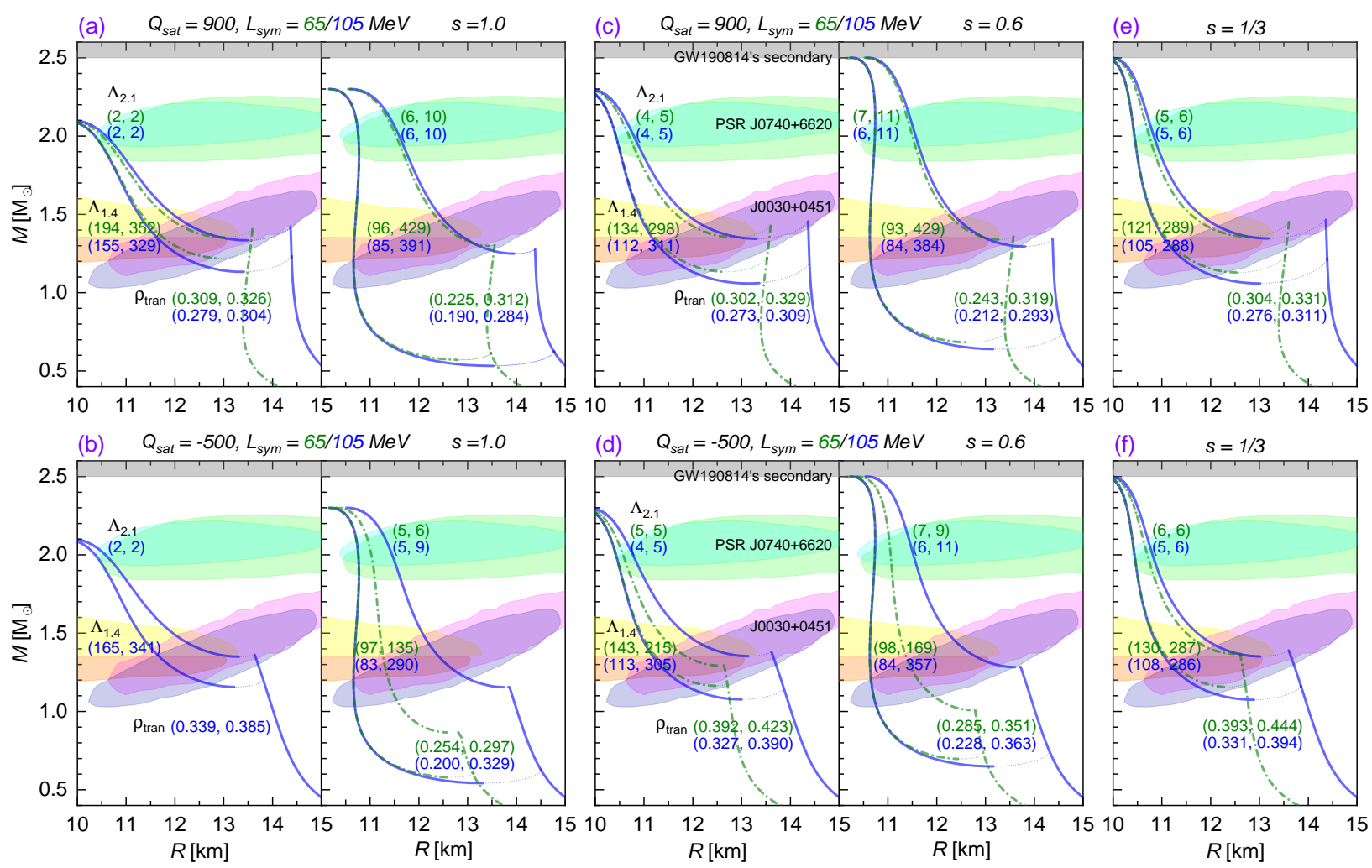

FIG. 3. Constraints on the $M-R$ relation of CSs featuring twin configurations (in some cases, the instability region is not resolved on the figure's scale). Panels (a) and (b): $M-R$ curves for hybrid stars characterized by different maximum masses (attained on the quark branch) for nucleonic EOSs with pairs of values of $Q_{\text {sat }}(-500$ and $900 \mathrm{MeV})$ and $L_{\mathrm{sym}}(65$ and $105 \mathrm{MeV})$, and quark-matter CSS EOSs with $s=1.0$ (in natural units). The ranges of transition density $\rho_{\text {tran }}$ in units of $\mathrm{fm}^{-3}$, and the TDs $\Lambda_{1.4}$ and $\Lambda_{2.1}$ (dimensionless) for a canonical mass $M / M_{\odot}=1.4$ and massive $M / M_{\odot}=2.1$ stars, respectively, are quoted as well. The color coding of numbers matches that of the curves. Panels (c), (d) and (e), (f): same as in (a), (b), but with $s=0.6$ and $s=1 / 3$, respectively.

(i.e., a discontinuity between the nuclear branch and the hybrid branch).

Let us first focus on the case $s=1.0$ [Figs. 3(a) and 3(b)]. The following systematics are observed in our setup:

(i). The stiffer is the nucleonic EoS the larger is the range of masses and radii where twin stars exist; see also Fig. 4 below. Intuitively, this makes sense, as the stiffer nuclear EoS pushes the nucleonic branch to higher radii, further from the hybrid branch.

(ii). The larger the value $M_{\max }^{\mathrm{Q}}$ the narrower the range of masses where twins exist and the instability region between nucleonic and hybrid stars. A higher value, in the current set-up, requires a smaller $\Delta \varepsilon$ to allow for a steep increase of the mass. Since for our parameter choice the $M-R$ curves pass through the GW170817 90\% credible region, the quoted values of TD are consistent with the upper limit set by the GW170817 on this quantity event [4].

Consider next the case of less stiff quark EOS with $s=$ 0.6 (Figs. 3(c) and 3(d)) and $s=1 / 3$ (Fig. 3(e) and 3(f)). The general features found above are replicated in this case as well. However, in this case we find twin solutions for models supporting heavier stars, with $M_{\max }^{\mathrm{Q}} / M_{\odot} \gtrsim 2.3$. Interestingly, the $M_{\max }^{\mathrm{Q}} / M_{\odot}=2.5$ sequence, in which the light component in the GW190814 would be a hybrid star (see Fig. 3), allows for twin solutions for $s=0.6,1 / 3$ in the considered range $65 \leq L_{\mathrm{sym}} \leq 105 \mathrm{MeV}$. Compared to the $s=1.0$ case for the same maximum mass $M_{\max }^{\mathrm{Q}}$ the value of $M_{\mathrm{max}}^{\mathrm{N}}$ is shifted upwards significantly, which promotes the appearance of twin configurations.

The analysis above shows, among other things, that NICER's 90\% CI for PSR J0740+6620 does not preclude a strong first-order phase transition, which (very robustly) leads to a shift of the radius toward smaller values compared to the case without phase transition. Furthermore, the fact that PREX-II analysis of Ref. [13] indicates $L_{\mathrm{sym}}>65 \mathrm{MeV}$ at a $68 \%$ confidence level and $L_{\text {sym }}>45 \mathrm{MeV}$ at $90 \%$ corroborates the scenario of such a strong first-order phase transition, as a large $L_{\text {sym }}$ allows for a large radius of the nucleonic lowmass stars and thus "leaves room" on the $M-R$ diagram for a hybrid branch with a smaller radius that is consistent with astrophysical measurements. In such a scenario, the appearance of twin configurations is possible. This leads us to the conclusion that even the relatively low-mass $M / M_{\odot} \leq 1.5 \mathrm{CSs}$ could be, in fact, hybrid stars.

Figure 4 shows the values of $M_{\max }^{\mathrm{N}}$ for which twins arise; the mass range of twins $\Delta M_{\text {twin }}$, defined as the range between $M_{\max }^{\mathrm{N}}$ and the minimum value of a CS mass on the hybrid branch; and the intervals of the radius $\Delta R_{\text {twin }}$ and TD $\Delta \Lambda_{\text {twin }}$ 

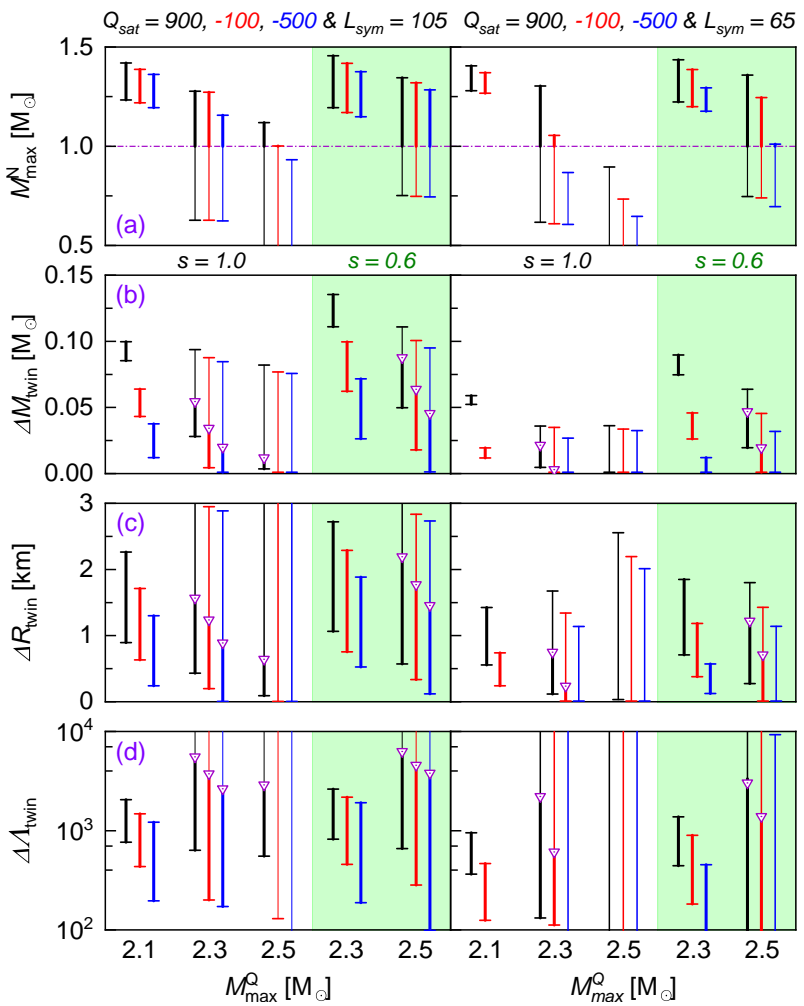

FIG. 4. Ranges of parameters $M_{\max }^{\mathrm{N}}, \Delta M_{\mathrm{twin}}, \Delta R_{\mathrm{twin}}$, and $\Delta \Lambda_{\mathrm{twin}}$ characterizing twin configurations (see text for definitions) for EOSs constructed using nucleonic matter with pairs of values of $Q_{\text {sat }}(-500$, -100 and $900 \mathrm{MeV})$ and $L_{\mathrm{sym}}(65$ and $105 \mathrm{MeV})$ and quark matter with $s=1.0$ and 0.6 (shadowed). The triangles followed by the thin lines correspond to twins with $M \leq 1.0 M_{\odot}$. See text for discussion.

associated with this mass range, as functions of the parameters defining the EOS. For $s=1.0$ twin configurations arise for $M_{\max }^{\mathrm{Q}} / M_{\odot} \lesssim 2.3$ (within the parameter space explored and excluding twins with $M<1.0 M_{\odot}$; see below), but for $s=0.6$, the values $M_{\max }^{\mathrm{Q}} / M_{\odot} \sim 2.5$ are obtained. This has implications for the GW190814 event, which involved a merger of a 23 $M_{\odot}$ black hole with a $\sim 2.6 M_{\odot}$ object. Therefore, this object could have been a hybrid star living on a CS sequence which contains a twin-i.e., the GW190814 event does not exclude twins. (For a contrary view see [56].)

In Fig. 4 we see that the range of masses containing twins is small, $\Delta M_{\text {twin }} \lesssim 0.05 M_{\odot}$. For radii, note that the triangles followed by thin lines denote twins with $M<1.0 M_{\odot}$. This is a rough threshold below which stars are unlikely to be observed because of their likely instability at the protoneutronstar stage $[57,58]$. With that condition, the radius difference of twins is $\Delta R_{\mathrm{twin}} \sim 1 \mathrm{~km}$ for $s=1.0$; the difference is larger for $s=0.6$ with $\Delta R_{\text {twin }}$ reaching up to $3 \mathrm{~km}$. This range is largest for very stiff nucleonic EOSs which predict a large radius. The narrow range of masses and the modest radius difference for twins is a consequence of the constraint imposed by the large radius of PSR J0740+662, which limits the allowed range of the reduction of the radius of a hybrid star and makes it challenging to distinguish between nucleonic and hybrid stars as $\Delta R_{\mathrm{twin}}=1 \mathrm{~km}$ requires radius measurement accuracy of less than $10 \%$. However, the difference in the TDs of twins can be several hundred to two thousand-see Fig. 4(d), therefore, studies of $\Lambda_{1}-\Lambda_{2}$ extracted from inspiral phase of CS mergers are a more promising avenue for identifying twins.

\section{CONCLUSIONS}

To summarize, we investigated the impact of two recent observational/experimental results, the inference of the radius of PSR J0740+6620 [2,3] from the x-ray data provided by the NICER experiment and that of the neutron skin thickness by the PREX-II experiment in conjunction with the DF analysis of Refs. [13, 14], on the static properties of relativistic hybrid stars. The analysis of Ref. [13] gives a value of $L_{\mathrm{sym}}$, which is in potential tension with the TD and radius limits inferred from the GW170817 analysis. The $L_{\mathrm{sym}}$ value extracted in the analysis of Ref. [14] is within the accepted range and does not pose any tension with multimessanger astrophysics. The above mentioned new results were confronted here with the conjecture of strong first-order phase transition and the formation of hybrid stars. In doing so, we adopted a densityfunctional model of nucleonic matter which allows for the accurate description of low-density nuclear phenomenology, along with a flexible parametrization of high-density quark matter. Our main finding is that it is possible to account for current astrophysical and nuclear experimental constraints within the scenario of hybrid stars, which can appear as twin configurations, with a low value of phase transition density from nucleonic to quark matter. Specifically, in this scenario, the first-order phase transition naturally softens the EOS at intermediate densities (which is required to avoid the potential tension between the GW170817 event and the PREX-II measurement's interpretation of Ref. [13]), and the assumption of a high sound speed in quark matter stiffens the EOS at high densities, which is required to account for $M \gtrsim 2.0 M_{\odot}$ massive CS and the large radius of PSR J0740+6620 (as measured by the NICER analysis teams). We have quantified under which conditions such models may have twin solutions. In particular, for the squared sound speed in quark matter $s=0.6$ and $s=1 / 3$, we find twin stars and a large enough maximum mass to allow for a CS in the GW190814 merger event. For low values of $s$, the range of the difference in the radii for twins expands; for example, $\Delta R_{\text {twin }} \sim 1.0 \mathrm{~km}$ found for $s=1$ becomes $3 \mathrm{~km}$ for $s=0.6$. In the EoS models where $\Delta$ resonances appear (see Refs. [30, 59] and references therein), the EOS softens at intermediate densities, and the radius of the star is reduced with consequences for the $M-R$ relation similar to those found here.

\section{ACKNOWLEDGMENTS}

M. A. is supported by the U.S. Department of Energy, Office of Science, Office of Nuclear Physics under Award No. DE-FG02-05ER41375. J. L. is supported by the "Funda- 
mental Research Funds for the Central Universities" under Grant No. SWU-020021. A. S. is supported by the Deutsche
Forschungsgemeinschaft (Grant No. SE 1836/5-1) and European COST Action "PHAROS" (CA16214).
[1] H. T. Cromartie, E. Fonseca, S. M. Ransom, et al. (NANOGrav), "Relativistic Shapiro delay measurements of an extremely massive millisecond pulsar," Nat. Astron. 4, 72-76 (2020), arXiv:1904.06759 [astro-ph.HE].

[2] T. E. Riley, A. L. Watts, P. S. Ray, et al., "A NICER View of the Massive Pulsar PSR J0740+6620 Informed by Radio Timing and XMM-Newton Spectroscopy," Astrophys. J. Lett. 918, L27 (2021), arXiv:2105.06980 [astro-ph.HE].

[3] M. C. Miller, F. K. Lamb, A. J. Dittmann, et al., "The Radius of PSR J0740+6620 from NICER and XMM-Newton Data," Astrophys. J. Lett. 918, L28 (2021), arXiv:2105.06979 [astroph.HE].

[4] B. P. Abbott, R. Abbott, T. D. Abbot, et al. (LIGO Scientific, Virgo), "Properties of the binary neutron star merger GW170817," Phys. Rev. X 9, 011001 (2019), arXiv:1805.11579 [gr-qc].

[5] Hung Tan, Travis Dore, Veronica Dexheimer, Jacquelyn Noronha-Hostler, and Nicolás Yunes, "Extreme Matter meets Extreme Gravity: Ultra-heavy neutron stars with crossovers and first-order phase transitions," arXiv eprints , arXiv:2106.03890 (2021), arXiv:2106.03890 [astro-ph.HE].

[6] Bhaskar Biswas, "Bayesian model-selection of neutron star equation of state using multi-messenger observations," arXiv eprints , arXiv:2106.02644 (2021), arXiv:2106.02644 [astroph.HE].

[7] Isaac Legred, Katerina Chatziioannou, Reed Essick, Sophia Han, and Philippe Landry, "Impact of the PSR J0740+6620 radius constraint on the properties of high-density matter," Phys. Rev. D 104, 063003 (2021), arXiv:2106.05313 [astro-ph.HE].

[8] G. Raaijmakers, S. K. Greif, K. Hebeler, T. Hinderer, S. Nissanke, A. Schwenk, T. E. Riley, A. L. Watts, J. M. Lattimer, and W. C. G. Ho, "Constraints on the dense matter equation of state and neutron star properties from NICER's mass-radius estimate of PSR J0740+6620 and multimessenger observations," Astrophys. J. Lett. 918, L29 (2021), arXiv:2105.06981 [astroph.HE].

[9] S. Huth, P. T. H. Pang, I. Tews, T. Dietrich, A. Le Fèvre, W. Trautmann, K. Agarwal, M. Bulla, M. W. Coughlin, and C. Van Den Broeck, "Constraining Neutron-Star Matter with Microscopic and Macroscopic Collisions," arXiv eprints , arXiv:2107.06229 (2021), arXiv:2107.06229 [nucl-th].

[10] Nai-Bo Zhang and Bao-An Li, "Impact of NICER's Radius Measurement of PSR J0740+6620 on Nuclear Symmetry Energy at Suprasaturation Densities," Astrophys. J. 921, 111 (2021), arXiv:2105.11031 [nucl-th].

[11] Shao-Peng Tang, Jin-Liang Jiang, Ming-Zhe Han, Yi-Zhong Fan, and Da-Ming Wei, "Constraints on the phase transition and nuclear symmetry parameters from PSR J0740+6620 and multimessenger data of other neutron stars," Phys. Rev. D 104, 063032 (2021), arXiv:2106.04204 [nucl-th].

[12] D. Adhikari, H. Albataineh, D. Androic, K. Aniol, D. S. Armstrong, et al. (PREX Collaboration), "Accurate determination of the neutron skin thickness of ${ }^{208} \mathrm{~Pb}$ through parity-violation in electron scattering," Phys. Rev. Lett. 126, 172502 (2021), arXiv:2102.10767 [nucl-ex].

[13] Brendan T. Reed, F. J. Fattoyev, C. J. Horowitz, and J. Piekarewicz, "Implications of PREX-2 on the equation of state of neutron-rich matter,' Phys. Rev. Lett. 126, 172503 (2021), arXiv:2101.03193 [nucl-th].

[14] Paul-Gerhard Reinhard, Xavier Roca-Maza, and Witold Nazarewicz, "Information content of the parity-violating asymmetry in ${ }^{208} \mathrm{~Pb}$," arXiv e-prints , arXiv:2105.15050 (2021), arXiv:2105.15050 [nucl-th].

[15] James M. Lattimer and Yeunhwan Lim, "Constraining the Symmetry Parameters of the Nuclear Interaction,” Astrophys. J. 771, 51 (2013), arXiv:1203.4286 [nucl-th].

[16] Pawel Danielewicz and Jenny Lee, "Symmetry energy II: Isobaric analog states," Nucl. Phys. A 922, 1-70 (2014), arXiv:1307.4130 [nucl-th].

[17] M. Oertel, M. Hempel, T. Klähn, and S. Typel, "Equations of state for supernovae and compact stars," Rev. Mod. Phys. 89, 015007 (2017), arXiv:1610.03361 [astro-ph.HE].

[18] M. Baldo and G. F. Burgio, "The nuclear symmetry energy," Prog. Part. Nucl. Phys. 91, 203-258 (2016), arXiv:1606.08838 [nucl-th].

[19] Mark G. Alford, Andreas Schmitt, Krishna Rajagopal, and Thomas Schäfer, "Color superconductivity in dense quark matter,” Rev. Mod. Phys. 80, 1455-1515 (2008), arXiv:0709.4635 [hep-ph].

[20] Eemeli Annala, Tyler Gorda, Aleksi Kurkela, and Aleksi Vuorinen, "Gravitational-wave constraints on the neutron-starmatter Equation of State,” Phys. Rev. Lett. 120, 172703 (2018), arXiv:1711.02644 [astro-ph.HE].

[21] Vasileios Paschalidis, Kent Yagi, David Alvarez-Castillo, David B. Blaschke, and Armen Sedrakian, "Implications from GW170817 and I-Love-Q relations for relativistic hybrid stars," Phys. Rev. D 97, 084038 (2018), arXiv:1712.00451 [astroph.HE].

[22] Elias R. Most, Lukas R. Weih, Luciano Rezzolla, and Jürgen Schaffner-Bielich, "New constraints on radii and tidal deformabilities of neutron stars from GW170817," Phys. Rev. Lett. 120, 261103 (2018), arXiv:1803.00549 [gr-qc].

[23] I. Tews, J. Margueron, and S. Reddy, "Critical examination of constraints on the equation of state of dense matter obtained from GW170817," Phys. Rev. C 98, 045804 (2018), arXiv:1804.02783 [nucl-th].

[24] G. F. Burgio, A. Drago, G. Pagliara, H. J. Schulze, and J. B. Wei, "Are Small Radii of Compact Stars Ruled out by GW170817/AT2017gfo?” Astrophys. J. 860, 139 (2018), arXiv:1803.09696 [astro-ph.HE].

[25] D. E. Alvarez-Castillo, D. B. Blaschke, A. G. Grunfeld, and V. P. Pagura, "Third family of compact stars within a nonlocal chiral quark model equation of state,” Phys. Rev. D 99, 063010 (2019), arXiv:1805.04105 [hep-ph].

[26] Jan-Erik Christian, Andreas Zacchi, and Jürgen SchaffnerBielich, "Signals in the tidal deformability for phase transitions in compact stars with constraints from GW170817," Phys. Rev. D 99, 023009 (2019), arXiv:1809.03333 [astro-ph.HE].

[27] Gloria Montana, Laura Tolos, Matthias Hanauske, and Luciano Rezzolla, "Constraining twin stars with GW170817," Phys. Rev. D 99, 103009 (2019), arXiv:1811.10929 [astro-ph.HE].

[28] M. Sieniawska, W. Turczanski, M. Bejger, and J. L. Zdunik, "Tidal deformability and other global parameters of compact stars with strong phase transitions," Astron. Astrophys. 622, 
A174 (2019), arXiv:1807.11581 [astro-ph.HE].

[29] Reed Essick, Philippe Landry, and Daniel E. Holz, "Nonparametric Inference of Neutron Star Composition, Equation of State, and Maximum Mass with GW170817," Phys. Rev. D 101, 063007 (2020), arXiv:1910.09740 [astro-ph.HE].

[30] Jia Jie Li, Armen Sedrakian, and Mark Alford, "Relativistic hybrid stars with sequential first-order phase transitions and heavy-baryon envelopes," Phys. Rev. D 101, 063022 (2020), arXiv:1911.00276 [astro-ph.HE].

[31] Zhiqiang Miao, Ang Li, Zhenyu Zhu, and Sophia Han, "Constraining hadron-quark phase transition parameters within the quark-mean-field model using multimessenger observations of neutron stars," Astrophys. J. 904, 103 (2020), arXiv:2006.00839 [nucl-th].

[32] Ang Li, Zhiqiang Miao, Sophia Han, and Bing Zhang, "Constraints on the maximum mass of neutron stars with a quark core from GW170817 and NICER PSR J0030+0451 data," Astrophys. J. 913, 27 (2021), arXiv:2103.15119 [astro-ph.HE].

[33] German Malfatti, Milva G. Orsaria, Ignacio F. Ranea-Sandoval, Gustavo A. Contrera, and Fridolin Weber, "Delta baryons and diquark formation in the cores of neutron stars," Phys. Rev. D 102, 063008 (2020), arXiv:2008.06459 [astro-ph.HE].

[34] M. C. Rodriguez, I. F. Ranea-Sandoval, M. Mariani, M. G. Orsaria, G. Malfatti, and O. M. Guilera, "Hybrid stars with sequential phase transitions: the emergence of the $\mathrm{g}_{2}$ mode," J. Cosm. Astro. Phys. 02, 009 (2021), arXiv:2009.03769 [astroph.HE].

[35] Mark G. Alford, Sophia Han, and Madappa Prakash, "Generic conditions for stable hybrid stars," Phys. Rev. D 88, 083013 (2013), arXiv:1302.4732 [astro-ph.SR].

[36] Mark G. Alford and Armen Sedrakian, "Compact stars with sequential QCD phase transitions," Phys. Rev. Lett. 119, 161104 (2017), arXiv:1706.01592 [astro-ph.HE].

[37] T. E. Riley, A. L. Watts, S. Bogdanov, et al., "A NICER View of PSR J0030+0451: Millisecond Pulsar Parameter Estimation," Astrophys. J. Lett. 887, L21 (2019), arXiv:1912.05702 [astroph.HE].

[38] M. C. Miller, F. K. Lamb, A. J. Dittmann, et al., "PSR J0030+0451 Mass and Radius from NICER Data and Implications for the Properties of Neutron Star Matter," Astrophys. J. Lett. 887, L24 (2019), arXiv:1912.05705 [astro-ph.HE].

[39] G. A. Lalazissis, T. Niksic, D. Vretenar, and P. Ring, "New relativistic mean-field interaction with density-dependent mesonnucleon couplings," Phys. Rev. C 71, 024312 (2005).

[40] Jia Jie Li and Armen Sedrakian, "Constraining compact star properties with nuclear saturation parameters," Phys. Rev. C 100, 015809 (2019), arXiv:1903.06057 [astro-ph.HE].

[41] Jérôme Margueron, Rudiney Hoffmann Casali, and Francesca Gulminelli, "Equation of state for dense nucleonic matter from metamodeling. II. Predictions for neutron star properties," Phys. Rev. C 97, 025806 (2018), arXiv:1708.06895 [nucl-th].

[42] E. Fonseca, H. T. Cromartie, T. T. Pennucci, et al., "Refined Mass and Geometric Measurements of the High-mass PSR J0740+6620," Astrophys. J. Lett. 915, L12 (2021), arXiv:2104.00880 [astro-ph.HE].

[43] Luciano Rezzolla, Elias R. Most, and Lukas R. Weih, "Using gravitational-wave observations and quasi-universal relations to constrain the maximum mass of neutron stars," Astrophys. J. Lett. 852, L25 (2018), arXiv:1711.00314 [astro-ph.HE].

[44] Sanika Khadkikar, Adriana R. Raduta, Micaela Oertel, and Armen Sedrakian, "Maximum mass of compact stars from gravi- tational wave events with finite-temperature equations of state," Phys. Rev. C 103, 055811 (2021), arXiv:2102.00988 [astroph.HE].

[45] R. Abbott, T. D. Abbott, S. Abraham, et al. (LIGO Scientific, Virgo), "GW190814: Gravitational Waves from the Coalescence of a 23 Solar Mass Black Hole with a 2.6 Solar Mass Compact Object,” Astrophys. J. Lett. 896, L44 (2020), arXiv:2006.12611 [astro-ph.HE].

[46] F. J. Fattoyev, C. J. Horowitz, J. Piekarewicz, and Brendan Reed, "GW190814: Impact of a 2.6 solar mass neutron star on the nucleonic equations of state," Phys. Rev. C 102, 065805 (2020), arXiv:2007.03799 [nucl-th].

[47] Jia Jie Li, Armen Sedrakian, and Fridolin Weber, "Rapidly rotating $\Delta$-resonance-admixed hypernuclear compact stars," Phys. Lett. B 810, 135812 (2020), arXiv:2010.02901 [astro-ph.HE].

[48] J. Estee, W. G. Lynch, C. Y. Tsang, et al. (S $\pi$ RIT Collaboration), "Probing the Symmetry Energy with the Spectral Pion Ratio,” Phys. Rev. Lett. 126, 162701 (2021), arXiv:2103.06861 [nucl-ex].

[49] Reed Essick, Philippe Landry, Achim Schwenk, and Ingo Tews, "A Detailed Examination of Astrophysical Constraints on the Symmetry Energy and the Neutron Skin of ${ }^{208} \mathrm{~Pb}$ with Minimal Modeling Assumptions,” Phys. Rev. Lett. 127, 192701 (2021), arXiv:2107.05528 [nucl-th].

[50] David Radice, Albino Perego, Francesco Zappa, and Sebastiano Bernuzzi, "GW170817: Joint Constraint on the Neutron Star Equation of State from Multimessenger Observations," Astrophys. J. Lett. 852, L29 (2018), arXiv:1711.03647 [astroph.HE].

[51] Kenta Kiuchi, Koutarou Kyutoku, Masaru Shibata, and Keisuke Taniguchi, "Revisiting the lower bound on tidal deformability derived by AT2017gfo," Astrophys. J. Lett. 876, L31 (2019), arXiv:1903.01466 [astro-ph.HE].

[52] J. L. Zdunik and P. Haensel, "Maximum mass of neutron stars and strange neutron-star cores," Astron. Astrophys. 551, A61 (2013), arXiv:1211.1231 [astro-ph.SR].

[53] Luca Bonanno and Armen Sedrakian, "Composition and stability of hybrid stars with hyperons and quark colorsuperconductivity," Astron. Astrophys. 539, A16 (2012), arXiv:1108.0559 [astro-ph.SR].

[54] D. Blaschke, T. Klahn, R. Lastowiecki, and F. Sandin, "How strange are compact star interiors ?" J. Phys. G 37, 094063 (2010), arXiv:1002.1299 [nucl-th].

[55] Mark G. Alford, Krishna Rajagopal, Sanjay Reddy, and Frank Wilczek, "Minimal color-flavor-locked-nuclear interface,” Phys. Rev. D 64, 074017 (2001), arXiv:hep-ph/0105009.

[56] Jan-Erik Christian and Jürgen Schaffner-Bielich, "Supermassive neutron stars rule out twin stars," Phys. Rev. D 103, 063042 (2021), arXiv:2011.01001 [astro-ph.HE].

[57] D. Gondek, P. Haensel, and J. L. Zdunik, "Radial pulsations and stability of protoneutron stars," Astron. Astrophys. 325, 217-227 (1997), arXiv:astro-ph/9705157.

[58] Klaus Strobel and Manfred K. Weigel, "On the minimum and maximum mass of neutron stars and the delayed collapse," Astron. Astrophys. 367, 582 (2001), arXiv:astro-ph/0012321.

[59] Armen Sedrakian, Jia-Jie Li, and Fridolin Weber, "Hyperonization in compact stars," arXiv e-prints , arXiv:2105.14050 (2021), arXiv:2105.14050 [nucl-th]. 